the oestradiol tablet experienced a meaningful benefit from treatment than those who had placebo.

In an accompanying commentary, Alison Huang and Deborah Grady state that the results of this study suggest that neither oestrogen tablets nor vaginal moisturizer offer a meaningful benefit for control of women's symptoms over placebo. These authors suggest that, on the basis of these findings, the current emphasis on oestrogen for treating vulvovaginal symptoms could be re-evaluated, owing to the lack of clear evidence to support the superiority of oestrogen-based therapy.

In conclusion, the results of this trial suggest that treatment is no better than placebo for vulvovaginal symptoms in postmenopausal women. Shared decision making should account for cost of therapy and patient preference.

Louise Stone

ORIGINAL ARTICLE Mitchell, C. M. et al. Efficacy of vaginal estradiol or vaginal moisturizer vs placebo for treating postmenopausal vulvovaginal symptoms: a randomized clinical trial. JAMA Intern. Med. https://doi.org/10.1001/jamainternmed. 2018.0116 (2018)

under nutrient-limiting conditions. "Knockout MEFs that were deficient in either ATG5 or PAK1 also enabled us to demonstrate that macropinocytosis trumps autophagy as a nutrient stress response," notes Edinger. "Cells can survive using autophagy but they can both survive and proliferate using macropinocytosis."

The authors showed that necrotic cell debris is taken up by prostate cancer cells by macropinocytosis. They also used patient samples and autochthonous tumour models to confirm that macropinocytosis occurred in primary prostate tumour samples and in a physiological context.

"Showing that AMPK activation can drive growth in low-nutrient medium by stimulating macropinocytosis adds to the growing body of literature suggesting that inhibiting - rather than activating - AMPK in established prostate tumours could be a superior therapeutic strategy," says Edinger.

Rebecca Kelsey

ORIGINAL ARTICLE Kim, S. M. et al. PTEN deficiency and AMPK activation promote nutrient scavenging and anabolism in prostate cancer cells. Cancer Discov. https://doi. org/10.1158/2159-8290.CD-17-1215 (2018)

\section{AR involved in sunitinib resistance}

New data, published in Cancer Research, show that androgen receptor (AR) expression and phosphorylation are increased in sunitinib-resistant kidney cancer. Combination treatment with the AR antagonist enzalutamide restored sensitivity to sunitinib in experimental models, providing a rationale for clinically testing this combination in sunitinib-resistant disease.

Integrative analysis of a reverse-phase protein array of a patient-derived xenograft (PDX) model of acquired sunitinib resistance showed increased AR expression. In vitro, kidney cancer cell lines with either acquired or intrinsic sunitinib resistance also showed increased AR expression. AR levels correlated with sunitinib sensitivity in AR-positive and AR-negative cell lines.

Overexpression of wild-type AR in AR-negative cells decreased sensitivity to sunitinib. Combined treatment of sunitinib and enzalutamide partially restored sunitinib sensitivity.

Combination treatment with sunitinib and enzalutamide had a synergistic effect in sunitinib-resistant, AR-positive cells, but enzalutamide alone did not significantly affect cell viability. Sunitinib treatment in AR-positive cells induced expression of $A R$ target genes, including $K L K 2$,

$K L K 4$, ZBTB16, and MYC, which co-treatment with enzalutamide inhibited. In culture supernatants, levels of KLK2 were increased in sunitinib-treated, sunitinib-resistant cells, but combination treatment with enzalutamide reduced these levels.

The absence of androgens in the culture media did not affect $A R$ expression or cell growth. Mass spectrometry of PDX models with acquired or intrinsic sunitinib resistance and kidney cancer cell lines did not show presence of considerable levels of androgens. Immunofluorescence showed increased AR nuclear translocation and phosphorylation of serine 81 in AR-positive cells after sunitinib treatment. Cyclin-dependent kinase 1 (CDK1), which phosphorylates serine 81 on the AR, showed increased expression in sunitinib-resistant models in vivo and in vitro on treatment with sunitinib.

Addition of a proteasome inhibitor to combination enzalutamide and sunitinib treatment abrogated enzalutamide-induced AR degradation in the presence of sunitinib. The addition of the proteasome inhibitor also increased cell proliferation. AR expression was increased by sunitinib treatment in AR-positive cells with transient Speckle-type

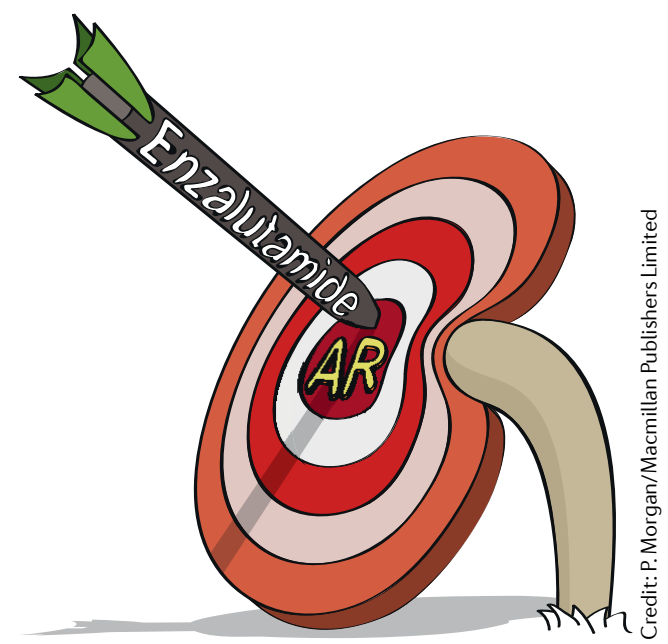

POZ protein (SPOP) knockdown. However, enzalutamide-induced AR degradation was inhibited in these cells in the presence of sunitinib treatment. Immunoprecipitation showed that ubiquitin associated with $A R$ after treatment with sunitinib in sunitinib-resistant cells. The reduction in AR nuclear translocation caused by enzalutamide was inhibited by competitive binding with dihydrotestosterone.

In vivo, combined sunitinib and enzalutamide treatment delayed time to acquired sunitinib resistance in sunitinib-sensitive, cell-line-derived xenografts. In experimental models with acquired sunitinib resistance, combination treatment inhibited further tumour growth compared with enzalutamide treatment alone. Circulating levels of KLK2 were increased in xenograft models of acquired and intrinsic sunitinib resistance.

In patients, serum KLK2 levels were increased in those whose disease progressed while receiving sunitinib plus dalteparin compared with those who did not experience disease progression.

Overall, these data suggest that the $A R$ is involved in sunitinib resistance in kidney cancer, which can be reversed by combination treatment with an AR antagonist. These data provide a rationale for clinically testing this combination in patients with sunitinib-resistant disease.

Louise Stone

ORIGINAL ARTICLE Adelaiye-Ogala, R. et al. Therapeutic targeting of sunitinib-induced AR phosphorylation in renal cell carcinoma. Cancer Res. https://doi.org/10.1158/0008-5472.CAN-17-3386 (2018) 